\title{
Nicousamide normalizes renovascular hypertension in two-kidney one-clip hypertensive rats
}

\author{
SEN ZHANG ${ }^{*}$, HONGYAN LI ${ }^{*}$, YAN LI, FURONG ZHANG, YUE LIU and XIAOGUANG CHEN \\ State Key Laboratory of Bioactive Substances and Functions of Natural Medicines, Institute of Materia Medica, \\ Chinese Academy of Medical Sciences and Peking Union Medical College, Beijing 100050, P.R. China
}

Received August 1, 2012; Accepted September 12, 2012

DOI: $10.3892 /$ br.2012.26

\begin{abstract}
Nicousamide, a coumarin-aspirin compound, was proven to have a renal protective effect on diabetic and hypertensive nephropathy. The present study aimed to investigate the anti-hypertensive effect of nicousamide and its action mechanisms. The two-kidney one-clip (2K1C) hypertensive animal model was introduced in this study. Subsequent to treatment with nicousamide for three weeks, systolic blood pressure (SBP) and diastolic blood pressure (DBP) were measured, and the plasma concentration of angiotensin II (Ang II), nitric oxide (NO), endothelin-1 (ET-1), nitric oxide synthase (NOS) and calcitonin gene-related peptide (CGRP) was examined. Results showed that nicousamide markedly decreased SBP as well as DBP on renovascular hypertensive rats $(\mathrm{P}<0.05)$. Nicousamide also reduced the Ang II and ET-1 concentration $(\mathrm{P}<0.05)$ in the plasma of hypertensive rats while increasing the plasma NO level $(\mathrm{P}<0.05)$. Nicousamide treatment did not show a marked effect on the NOS and CGRP concentrations in animal plasma $(\mathrm{P}>0.05)$.
\end{abstract}

\section{Introduction}

Nicousamide, a novel coumarin-aspirin compound, has been categorized as a 1.1 class drug and is undergoing stage I clinical trials in China (1). Its structure is shown in Fig. 1. Previous studies have proven its potential to prevent diabetic nephropathy in rats via the inhibition of advanced glycation end products (ACEs) (2,3). Besides inhibiting ACEs formation, nicousamide is a potent inhibitor of the phosphorylation of

Correspondence to: Professor Xiaoguang Chen, State Key Laboratory of Bioactive Substances and Functions of Natural Medicines, Institute of Materia Medica, Chinese Academy of Medical Sciences and Peking Union Medical College, Beijing 100050, P.R. China

E-mail: chxg@imm.ac.cn

${ }^{*}$ Contributed equally

Key words: nicousamide, hypertension, two-kidney one-clip, angiotensin II, nitric oxide, endothelin-1
TGF- $\beta$ receptor II (1). By blocking the TGF- $\beta$-Smad signaling pathway, nicousamide retards renal fibrogenesis and exhibits a renal protective effect.

Recently, we also demonstrated that nicousamide is able to suppress hypertensive nephropathy in spontaneously hypertensive rats (SHRs) during the long-term treatment (data under publication), although its mechanism remains unclear. Nicousamide shows a marked anti-hypertensive effect in SHRs, while its effect is weaker compared to losartan. In the present study, we examined its mechanism on blood pressure (BP) control, by introducing the two-kidney one-clip (2K1C) model of hypertension in order to investigate its anti-hypertension action. Additionally, by analyzing angiotensin II (Ang II), nitric oxide (NO), endothelin-1 (ET-1), nitric oxide synthase (NOS) and calcitonin gene-related peptide (CGRP) levels in the plasma of the model animals, we aimed to understand the underlying mechanisms by which nicousamide reduces BP. Captopril was also included in the study as the positive control to better evaluate the potency of nicousamide.

\section{Materials and methods}

Animals. Six-week-old adult male Wistar rats, weighing 160-180 g, were purchased from the Institute of Laboratory Animal Science, Chinese Academy of Medical Sciences (Beijing, China). They were kept in a specific-pathogen-free facility with food and water ad libitum, at a constant temperature $\left(22 \pm 2^{\circ} \mathrm{C}\right)$ and a daily illumination period of $12 \mathrm{~h}$ (7:00 a.m.-7:00 p.m.). The study was approved by our Institutional Animal Care Committee of China, and the procedures were in accordance with the National Institute of Health Guide for the Care and Use of Laboratory Animals.

Reagents and drugs. Nicousamide was synthesized by the Department of Medicinal Chemistry, Institute of Materia Medica, Chinese Academy of Medical Science (Beijing, China), with $99 \%$ purity by high performance liquid chromatography (HPLC). Its chemical structure is shown in Fig. 1. Captopril was purchased from Beijing Taiyang Pharmaceutical Industry Co., Ltd. (Beijing, China).

Induction of renovascular hypertension, grouping and treatment. Induction of renal hypertension was carried out in rats according to the $2 \mathrm{~K} 1 \mathrm{C}$ model modified by previous studies 
$(4,5)$. Briefly, rats were anaesthetized with $3 \%$ sodium pentobarbital [ $30 \mathrm{mg} / \mathrm{kg}$ by intraperitoneal injection (i.p.)]. The left posterior side of the animal was shaved and sterilized with $70 \%$ ethanol. An $\sim 2 \mathrm{~cm}$ incision was made subcutaneously through the muscles just below the ribs. The left kidney was then exposed and retracted to expose the renal artery. A silver clip with an internal diameter of 0.15-0.20 mm was placed around the left renal artery as close as possible to the aorta. The clip was then placed with the slit opening facing the abdomen. The contralateral kidney was left intact. The muscles and skin were sutured and the rats were allowed to recover from anaesthesia and left for six weeks to develop hypertension. During this time period, SBP and the heart rate (HR) were measured at weekly intervals. Sham-operated rats underwent the same surgical procedure except for the clip placement. After six weeks, only animals with SBP $>160 \mathrm{mmHg}$ were selected for further drug administration. The BP and HR was measured by tail-cuff plethysmography (BP-98A; Softron, Tokyo, Japan) with prior training to minimize variability in the BP measurement.

The hypertensive rats were randomized into five groups: the sham and model controls, captopril $[6 \mathrm{mg} / \mathrm{kg}$, periocularly (p.o.)], and nicousamide (30 and $60 \mathrm{mg} / \mathrm{kg}$, p.o.) groups. Administration continued for three weeks and systolic blood pressure (SBP), diastolic blood pressure (DBP) and HR were measured at the end of treatment.

Preparation of blood samples for biochemical analysis. Subsequent to 3-week nicousamide treatment, blood of rats was sampled through the eyes after rats were anesthetized with diethyl ether. Blood plasma was obtained for biochemical analysis. Blood samples $(3 \mathrm{ml})$ were placed in tubes containing $1 \%$ heparin $(0.1 \mathrm{ml})$. Plasma was obtained by centrifugation at $1200 \mathrm{x} \mathrm{g}$ for $20 \mathrm{~min}\left(4^{\circ} \mathrm{C}\right)$.

Determination of NO level. The total amount of NO (NOx) was indirectly estimated in terms of its main metabolites, nitrate and nitrite by the Griess reaction after the sample was de-proteinized with absolute ethanol. The absorbance was measured at $540 \mathrm{~nm}$ and the results were expressed in the serum samples as $\mu \mathrm{mol} / 1$.

Determination of Ang II, ET-1, NOS and CGRP levels in rat plasma. The plasma concentration of Ang II was determined by Ang II radioimmuoassay kits (Beijing North Institute of Biological Technology, Beijing, China) (6), and ET-1 level in plasma was measured by a colorimetric immunometric enzyme immunoassay kit (IBL, Tokyo, Japan). Determination of the total NOS concentration in plasma was determined using the ELISA kit (Biomart, Shanghai, China). CGRP was determined using the ELISA kit (Cayman Chemical, Michigan, USA), The detailed procedure was carried out following the manufacturer's instructions.

Statistical analysis. Data were expressed as the mean \pm SEM. A comparison between the mean values of different groups was carried out using one-way analysis of variance (ANOVA), followed by the Tukey-Kramer post hoc test for multiple comparisons. In the data analysis, $\mathrm{P}<0.05$ was considered to indicate a statistically significant difference.

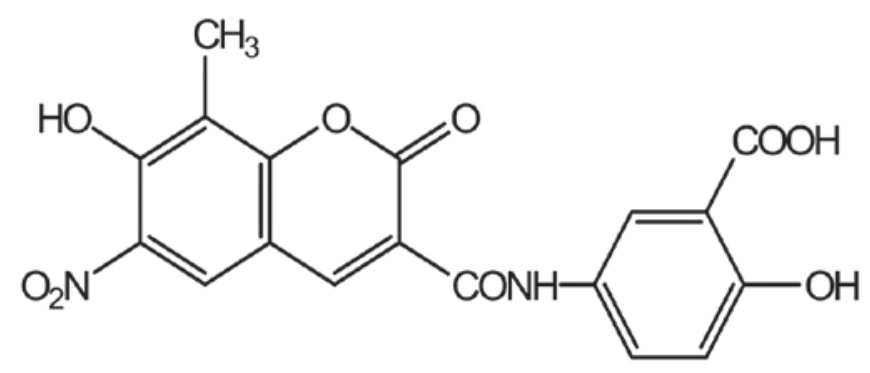

Figure 1. Chemical structure of nicousamide.

\section{Results}

Nicousamide decreased the SBP and DBP in 2 K1C hypertensive rats. Six weeks after $2 \mathrm{~K} 1 \mathrm{C}$ operation, the hypertensive rats (SBP >160 mmHg) were enrolled in the treatment. As shown in Table I, after three weeks of treatment, the model group still had a markedly higher average DBP and SBP compared to the sham group $(\mathrm{P}>0.05)$. Simultaneously, nicousamide treatment reduced the SBP by $\sim 9 \%$ at two doses, compared to the model control $(\mathrm{P}<0.05)$, and similar to captopril, nicousamide also markedly reduced DBP. However, nicousamide did not exhibit a statistically significant effect on $\mathrm{HR}$ in $2 \mathrm{~K} 1 \mathrm{C}$ hypertensive rats.

Nicousamide decreased Ang II and ET-1 levels in the plasma of renovascular hypertensive rats. As shown in Table II, nicousamide treatment decreased the plasma Ang II level in $2 \mathrm{~K} 1 \mathrm{C}$ rats by 29 and $37 \%$, respectively, at the doses of 30 and $60 \mathrm{mg} / \mathrm{kg}(\mathrm{P}<0.05)$, compared to the model control. This treatment therefore had a better effect compared to captopril.

Compared to the model control, plasma ET-1 concentration in nicousamide-treated groups was reduced by $\sim 21.5 \%$ at 30 and $60 \mathrm{mg} / \mathrm{kg}$, which was less effective compared to captopril.

Nicousamide increased NO level in plasma, but had no effect on NOS. As shown in Table II, nicousamide increased the plasma NO concentration in renovascular hypertensive rats at two doses. In order to understand the underlying mechanisms by which nicousamide reduced the NO level, the total NOS level in the plasma was tested, and no statistically significant difference was detected in the model group and the nicousamide-treated groups.

Nicousamide had no effect on plasma CGRP levels. Due to large standard deviation (SD), no statistically significant difference was observed in CGRP concentration among the five experimental groups, however, additional investigation is required.

\section{Discussion}

Previous studies have shown nicousamide to have a beneficial effect on hypertensive nephropathy in SHRs, correlated with anti-hypertension and the downregulation of Ang II (data under publication). To confirm that nicoumisade reduces BP, 
Table I. Values of SBP, DBP and HR after 3-week administration of nicousamide and captopril.

\begin{tabular}{lccr}
\hline Groups & SBP $(\mathrm{mmHg})$ & DBP $(\mathrm{mmHg})$ & HR $(\mathrm{beats} / \mathrm{min})$ \\
\hline Sham control & $154.1 \pm 9.14$ & $125.7 \pm 15.79$ & $331.6 \pm 44.9$ \\
Model control & $172.91 \pm 10.17^{\mathrm{a}}$ & $145.7 \pm 12.82^{\mathrm{a}}$ & $354.6 \pm 29.76$ \\
Captopril $(6 \mathrm{mg} / \mathrm{kg})$ & $157.8 \pm 11.74^{\mathrm{c}}$ & $130.3 \pm 15.41^{\mathrm{b}}$ & $355.4 \pm 32.74$ \\
Nicousamide $(30 \mathrm{mg} / \mathrm{kg})$ & $158.3 \pm 14.46^{\mathrm{b}}$ & $125.9 \pm 12.27^{\mathrm{c}}$ & $362.7 \pm 32.51$ \\
Nicousamide $(60 \mathrm{mg} / \mathrm{kg})$ & $158.1 \pm 18.87^{\mathrm{b}}$ & $127.8 \pm 26.97$ & $336.1 \pm 58.38$ \\
\hline
\end{tabular}

${ }^{\mathrm{a}} \mathrm{P}<0.05$ vs. sham control; ${ }^{\mathrm{b}} \mathrm{P}<0.05,{ }^{\mathrm{c}} \mathrm{P}<0.01$ vs. model control. SBP, systolic blood pressure; DBP, diastolic blood pressure; HR, heart rate.

Table II. Values of Ang II, ET-1, NO, NOS and CGRP after 3-week administration of nicousamide.

\begin{tabular}{|c|c|c|c|c|c|}
\hline Groups & Sham control & Model control & Captopril & $\begin{array}{l}\text { Nicousamide } \\
(30 \mathrm{mg} / \mathrm{kg})\end{array}$ & $\begin{array}{c}\text { Nicousamide } \\
(60 \mathrm{mg} / \mathrm{kg})\end{array}$ \\
\hline Ang II (pg/ml) & $80.7 \pm 14.9$ & $139.3 \pm 11.1^{\mathrm{a}}$ & $130.6 \pm 53.6$ & $99.2 \pm 53.2^{b}$ & $87.4 \pm 28.9^{\mathrm{b}}$ \\
\hline ET-1 (pg/ml) & $176 \pm 87$ & $331 \pm 190^{\mathrm{a}}$ & $205 \pm 88$ & $260 \pm 116^{\mathrm{b}}$ & $264 \pm 129^{b}$ \\
\hline $\mathrm{NO}(\mu \mathrm{mol} / \mathrm{l})$ & $13.3 \pm 9.4$ & $5.2 \pm 2.4^{\mathrm{a}}$ & $10.2 \pm 7.4$ & $9.6 \pm 5.5$ & $13.6 \pm 9.6^{b}$ \\
\hline NOS (U/mg prot) & $26.6 \pm 3.0$ & $26.1 \pm 2.7$ & $24.4 \pm 3.2$ & $20.9 \pm 7.5$ & $24.3 \pm 4.1$ \\
\hline CGRP (pg/ml) & $51 \pm 31$ & $40 \pm 20$ & $38 \pm 25$ & $35 \pm 30$ & $49 \pm 27$ \\
\hline
\end{tabular}

${ }^{\mathrm{a}} \mathrm{P}<0.05$ vs. sham control; ${ }^{\mathrm{b}} \mathrm{P}<0.05$ vs. model control. Ang II, angiotensin II; ET-1, endothelin-1; NO, nitric oxide; NOS, nitric oxide synthase; CGRP, calcitonin gene-related peptide.

the $2 \mathrm{~K} 1 \mathrm{C}$ renovascular hypertensive rodent model was introduced in the present study. The $2 \mathrm{~K} 1 \mathrm{C}$ rat model of arterial hypertension is well-established and reflects a common condition in a large number of humans with secondary forms of hypertension, thus, it is a renovascular Ang II-dependent form of arterial hypertension $(7,8)$.

After 2K1C operation for six weeks, we detected a marked increase in the SBP and DBP in all the groups, with the exception of the sham group, demonstrating the successful establishment of a renovascular hypertension model. After a three-week oral administration of nicousamide and captopril, we further confirmed that nicousamide had a marked anti-hypertensive effect, on SBP as well as DBP, and its effect was almost identical to that of captopril. No notable changes were observed in HR in any of the groups.

To understand the underlying mechanisms of the antihypertensive effect, the plasma concentration in Ang II, NO, ET-1 and CPRP was examined. Ang II was found to be a strong vasoconstrictor. In the present study, nicousamide treatment markedly reduced the Ang II level in a dose-dependent manner, suggesting that nicousamide may reduce the BP via the suppression of plasma Ang II.

Accumulating evidence suggests that NO plays a significant role in the regulation of $\mathrm{BP}$ and that impaired $\mathrm{NO}$ bioactivity is an important component of hypertension (9). Mice with disruption of the gene for endothelial NOS (eNOS) exhibit elevated BP levels compared to the control animals, suggesting a NOS to the link between impaired NO bioactivity and hypertension (10). In the current study, the plasma NO level in the $2 \mathrm{~K} 1 \mathrm{C}$ model rats was markedly decreased by $60.9 \%$ compared to the sham control. Notably, nicousamide treatment could raise the plasma NO level in hypertensive rats in a dose-dependent manner, thus, at a dose of $60 \mathrm{mg} / \mathrm{kg}$, the NO level is almost identical to that of the sham control, thereby explaining its anti-hypertension effect. To further investigate this mechanism, we examined the total NOS concentration in the animal plasma, including eNOS and inducible NOS (iNOS). However, the nicousamide dose did not have a statistically significant effect on NOS. These results suggest that nicousamide reduces the plasma NO concentration in hypertensive rats, although the detailed mechanism remains to be elucidated.

ET-1 is a paracrine or autocrine hormone, which was characterized as a vasoconstrictor and is overexpressed in the vasculature and blood in various models of hypertension, including the $2 \mathrm{~K} 1 \mathrm{C}$ model (11). In the present study, treatment using nicousamide downregulates the ET-1 level in the plasma of hypertensive rats, suggesting the presence of another action mechanisms of anti-hypertension.

We also examined another potent peptide vasodilator, CGRP. In subtotal nephrectomy (SN) and salt-induced hypertension, CGRP plays a compensatory role to attenuate the BP increase in the absence of an increase in the neuronal synthesis and release of this peptide $(12,13)$. Therefore, the purpose of this study was to determine whether or not the mechanism of this anti-hypertensive activity is through enhanced sensitivity of the vasculature to the dilator actions of this neuropeptide. However, preliminary results suggest that nicousamide did not markedly affect the plasma CGRP concentration. 
In summary, nicousamide has a beneficially anti-hypertensive effect in $2 \mathrm{~K} 1 \mathrm{C}$ rat model, its mechanism possibly correlated with Ang II and ET-1 suppression, and the increase of plasma NO concentration. However, the detailed mechanisms requires further investigation.

\section{Acknowledgements}

The authors would like to thank the Key Project of the National Eleventh-Five Year Research Program of China, for their support.

\section{References}

1. Zhang H-j, Jin J, Zhou W-q, et al: Nicousamide, a potent inhibitor of phosphorylation by TGF- $\beta$ receptor II. Acta Pharm Sinica B1: 160-165, 2011.

2. Li H, Zhang Y, Wang H, Zheng $X$ and Chen X: Nicousamide blocks the effects of advanced glycation end products on renal cells. Eur J Pharmacol 674: 455-459, 2012.

3. Li H, Zheng X, Wang H, Zhang Y, Xin H and Chen $\mathrm{X}$ : XLF-III-43, a novel coumarin-aspirin compound, prevents diabetic nephropathy in rats via inhibiting advanced glycation end products. Eur J Pharmacol 627: 340-347, 2010.

4. Laffan RJ, Goldberg ME, High JP, Schaeffer TR, Waugh MH and Rubin B: Antihypertensive activity in rats for SQ 14,225, an orally active inhibitor of angiotensin I-converting enzyme. J Pharmacol Exp Ther 204: 281-288, 1978.
5. Mansour SM, Bahgat AK, El-Khatib AS and Khayyal MT: Ginkgo biloba extract (EGb 761) normalizes hypertension in $2 \mathrm{~K}, 1 \mathrm{C}$ hypertensive rats: role of antioxidant mechanisms, ACE inhibiting activity and improvement of endothelial dysfunction. Phytomedicine 18: 641-647, 2011.

6. Lin L, Lv S and Li B: Angiotensin-I-converting enzyme (ACE)-inhibitory and antihypertensive properties of squid skin gelatin hydrolysates. Food Chem 131: 225-230, 2012.

7. Nishi EE, Campos RR, Bergamaschi CT, de Almeida VR and Ribeiro DA: Vitamin C prevents DNA damage induced by renovascular hypertension in multiple organs of Wistar rats. Hum Exp Toxicol 29: 593-599, 2010.

8. DiBona GF: Nervous kidney: interaction between renal sympathetic nerves and the renin-angiotensin system in the control of renal function. Hypertension 36: 1083-1088, 2000.

9. Hermann M, Flammer A and Lüscher TF: Nitric oxide in hypertension. J Clin Hypertens 8: 17-29, 2006.

10. Huang PL, Huang Z, Mashimo $\mathrm{H}$, et al: Hypertension in mice lacking the gene for endothelial nitric oxide synthase. Nature 377: 239-242, 1995.

11. Iglarz M and Schiffrin E: Role of endothelin-1 in hypertension. Curr Hypertens Rep 5: 144-148, 2003.

12. Supowit SC, Katki KA, Hein TW, et al: Vascular reactivity to calcitonin gene-related peptide is enhanced in subtotal nephrectomy-salt induced hypertension. Am J Physiol Heart Circ Physiol 301: H683-H688, 2011.

13. Smillie SJ and Brain SD: Calcitonin gene-related peptide (CGRP) and its role in hypertension. Neuropeptides 45: 93-104, 2011. 